\section{ACUTE INVERSION OF THE UTERUS} BY

J. V. O'SULLIVAN, M.D., M.A.o., F.R.C.O.G., F.R.C.S. Obstetric Surgeon, City of London Maternity, Queen Mary's, and Bearsted Memorial Hospitals; Obstetric and Gynaecological Surgeon, Kingston County Hospital

The object of this paper is to draw attention to a simple method of replacement of the acutely inverted uterus which my colleagues and I have not heard of to date-to wit, intravaginal hydraulic pressure attained in a very simple manner. This succeeded in the following two cases.

\section{Case I}

Mrs. A., aged 31; first pregnancy, 1938-normal labour and puerperium; infant $6 \mathrm{lb} .8 \mathrm{oz}$. The second pregnancy was normal; the expected date was given as early February. On Feb. 15, 1945, surgical induction by Drew Smythe catheter was performed. Spontaneous delivery of a living child weighing $6 \mathrm{lb} .12 \mathrm{oz}$. occurred 12 hours later. The first and second stages of labour together lasted

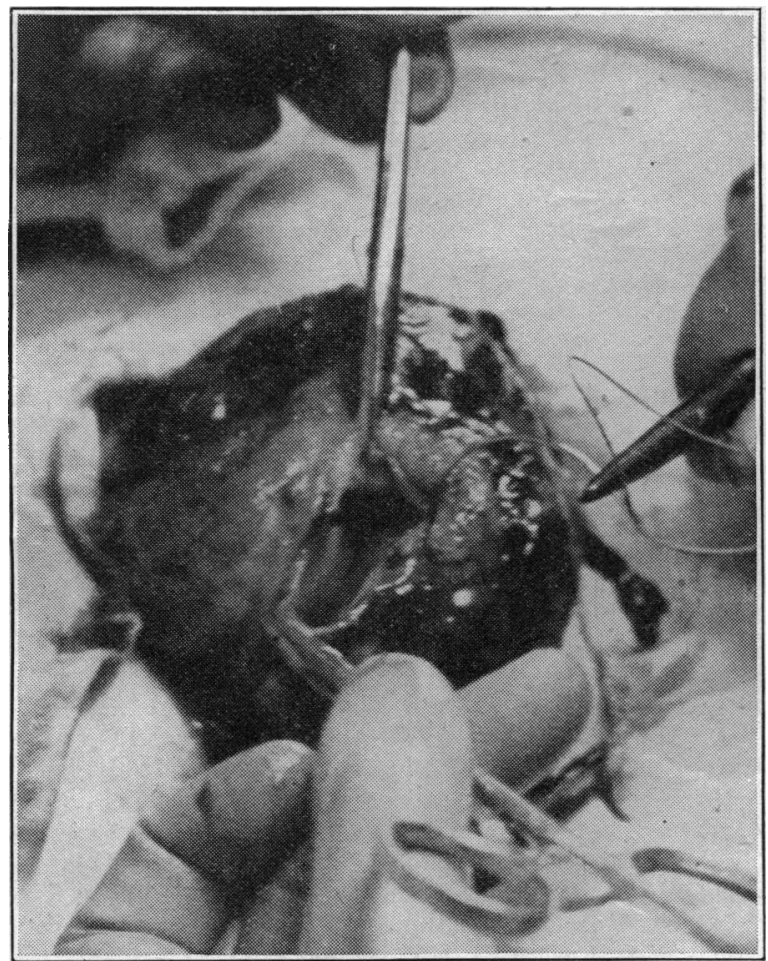

FIG. 1.-Case II. Inverted uterus, showing complete rupture.

three hours. There was moderate haemorrhage, so Crede's expression was practised, and immediately the uterus became completely inverted, the separated placenta being delivered at the same time. The uterus was replaced in the vagina at once, but further manipulation was considered inadvisable in view of the patient's shocked condition. I saw her 1 hour and 10 minutes after the inversion. She was pale and sweating; B.P. 80/50 and pulse 140. On palpating the abdomen no fundus uteri could be felt, and on parting the labia the red fundus uteri could be seen with the placental site on the fundus. Treatment for shock included three pints of blood. Three hours later the patient's general condition had greatly improved, so open ether was given and replacement by taxis was attempted. This manœuvre caused a rapid deterioration in her condition, so I decided to give a douche preparatory to a later attempt. In carrying out the douche the tip of the nozzle, guarded by my fingers, was passed towards the posterior fornix; my forearm happened to block the vaginal orifice and the vagina ballooned out, and to my surprise and pleasure the uterus promptly returned to its correct position, the signs of shock rapidly disappeared, and the patient made an uninterrupted recovery.

\section{Case II}

Mrs. B., aged 24 ; an especially interesting case, as she had had acute inversion with her second confinement. In 1941 the first pregnancy and labour were normal apart from mild toxaemia towards the end of pregnancy. In 1942, with her second, she had a normal ante-natal history. On Oct. 4, 1942, after a surgical induction on the same day, she had an easy spontaneous delivery of a female child weighing $7 \mathrm{lb} .3 \mathrm{oz}$. There was an adherent placenta, and after blood-grouping she was given a general anaesthetic, when the placenta was found to be adherent to an inverted uterus. The placenta was peeled off. The patient at the end of this operation was so shocked that no attempt could be made at replacement of the uterus. She was given a blood transfusion and gradually improved. The next day her haemoglobin was only $52 \%$. She was given sulphonamides prophylactically. On Nov. 8 an Aveling's repositor was introduced and the uterus slowly and successfully replaced.

In her third pregnancy Mrs. B. attended at 36 weeks with the breech presenting; external version failed to correct the presentation. The pregnancy remained normal and the patient went into spontaneous labour on April 23, 1945, and had an easy breech delivery of a female child weighing $7 \mathrm{lb}$. $2 \mathrm{oz}$., alive and well. When the placenta was thought to have separated from the uterus an attempt was made to express it by fundal pressure and traction on the cord. Inversion of the uterus followed at once. As the patient was bleeding, an attempt to strip off the placenta was made, and this resulted in two large tears-one near the fundus, 2 in. long, through all three layers; the second on the left side, 1 in. long,

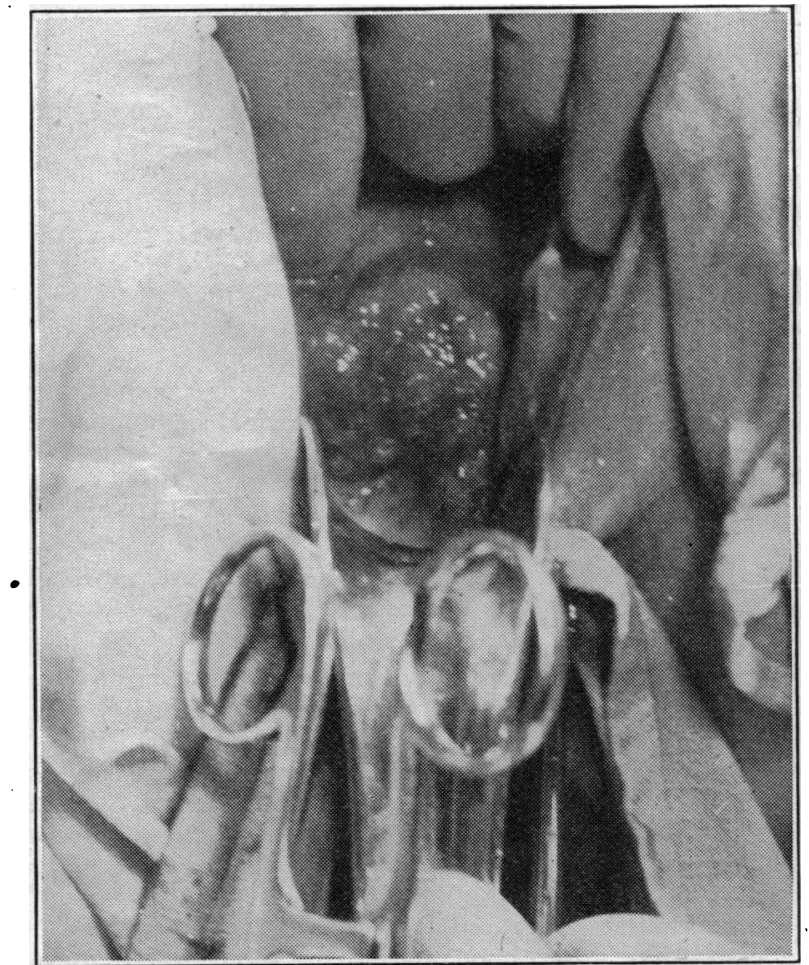

Fig. 2.-Case II. Cervix after replacement of inverted uterus by ballooning vagina, etc., by sterile douche.

also complete. At this stage the patient was desperately ill, with B.P. $70 / 40$ and pulse 160 . The placenta was finally strippedbleeding being controlled by ergometrine and hot packs-the uterus dusted with sulphanilamide powder, and replaced in the vagina. Blood drip, 2 pints, was given, then reconstituted human serum, 2 pints. I saw this patient about 9 hours after the inversion, when she complained of backache and severe pain in her left shoulder. Her condition had improved sufficiently to warrant further treatment, though no major surgery could yet be attempted, her B.P. being $110 / 75$ and pulse 120 . Light open ether anaesthesia was given. The uterus was gently delivered through the vagina. The adjacent peritoneal cavity tubes and ovaries were inspected through the tears and found normal. The two large tears were sewn up in two layers-continuous catgut for the peritoneum and interrupted catgut for the muscle layer, which was found to be thin over the lateral tear.

The uterus was douched with proflavine and dettol, then dusted with sulphanilamide powder and again replaced in the vagina. Gentle taxis failed to replace the uterus and increased the pulse from 120 to 150 ; the B.P. also began to fall. At this stage, in spite. of the ruptured uterus, which had been firmly sutured, it was decided to make an attempt to replace with hydraulic pressure as in the previous case. The vagina was distended to capacity by means of a dettoland-proflavine douche, retained by blocking the vaginal outlet with my forearm, aided by my assistant's (Dr. England's) hands. The 
uterus went back promptly and smoothly, the douche-can being held two to three feet above the vagina. The accompanying photographs, taken at the time, show (Fig. 1) the inverted uterus, with one of the complete tears; (Fig. 2) the cervix after replacement.

At the end of the operation there was no clinical evidence of any free fluid in the peritoneal cavity and the patient's condition was fair. Her puerperium was febrile for two weeks, but ultimately she made a complete recovery under first sulphanilamide then penicillin treatment.

\section{Synopsis of the Complication}

Acute inversion of the uterus means a rapid turning of the uterus inside out per vias naturales. This serious complication usually occurs during the third stage, though sometimes it may begin towards the end of the second stage by the pull on a short cord or soon after the separation of the placenta. Various authors put the incidence at between 1 in 200,000 and 1 in 17,000 deliveries. It usually occurs after a full-term labour, but has been reported in a five-months miscarriage.

The condition may be divided into (a) incomplete-including all degrees from a placental dimple to inversion into the vagina ; (b) complete-where the fundus is outside the vagina. The common cause is mismanagement of the third stage of labour by : (1) pulling on the cord when the uterus is relaxed ; (2) pushing on the fundus of a relaxed uterus ; (3) a combination of these two. In the two cases just described one or both were caused by one of these three ways. The catastrophe is most likely to occur if the placenta is attached to the fundus. Careful records of this point would be of great help. Wellauthenticated cases of spontaneous inversion have been reported.

The symptoms are usually very severe shock, out of all proportion to the amount of blood lost ; a tendency to bearing down after the placenta has been delivered; and, later in the puerperium, a persistent red haemorrhage or oft-repeated small haemorrhages, with obvious signs of anaemia and difficulty in micturition.

The signs are as follows. A complete inversion is self-evident. An incomplete inversion may be mistaken for a large fibroid polyp, but if the bladder is empty an abdominal examination will reveal the absence of the fundus uteri in the abdomen, and in its place a saucer-shaped depression where the fundus has been invaginated into the uterine cavity. Examination reveals a large rounded polypoid mass with a broad pedicle in the vagina. This, with the abdominal findings, renders the diagnosis obvious."

The prognosis is always very grave, the mortality varying between 23 and $80 \%$ in different series. If left alone, sloughing and/or sepsis may occur. In some cases spontaneous reinversion in a matter of hours, in others even during the passage of years, has been reported.

Treatment.-(1) Immediate replacement as soon as the inversion occurs, and before shock develops, without attempting to strip the placenta if it is still attached. (2) If the patient is very shocked, first clean the uterus and change the complete inversion into an incomplete by gently replacing the inverted uterus in the vagina, then raise the foot of the bed, put a sterile pad on the vulva, and carry out treatment for shock. (3) When shock is controlled replace the uterus as described above by hydraulic pressure.

My grateful thanks are due to Dr. H. R. England and Dr. H. Mayer for their great help in the second case, and to Dr. L. B. Bourne for the excellent photographs.

Writing on vision and selection of personnel in industry in the July/August number of Industrial. Welfare and Personnel Management Mr. Joseph Minton, F.R.C.S., expresses concern at the haphazard methods of vision testing in industry, many firms insisting on visual standards which are too high, others having no standards at all. As he says, men and women with all grades of vision can be employed in industry to-day, and he gives a chart for use as a general guide when selecting workers. In the same issue is an account by Col. S. D. Large of the planned exercises, mental and physical, at an Army convalescent depot which enables $85 \%$ of the men to leave fit. From his experience 70,000 cases Col. Large concludes that the principles practised at this depot could be applied with gain in civil centres. Dr. J. B. L. Tombleson describes a scheme operating in Bedford which provides a medical service to $10 \mathrm{firms}$ employing about 7,000 workers.

\section{ADMINISTRATION OF PENICILLIN BY INTRAMUSCULAR INFUSION}

BY

\section{E. C. TURTON,* M.B., B.S.}

(The Radcliffe Penicillin Unit of the Medical Research Council)

This account deals solely with the clinical aspects of the administration of penicillin by intramuscular infusion as described by Morgan, Christie, and Roxburgh (1944). Experience with 30 patients is summarized and attention is principally focused on three points: (i). whether this method was preferred by the patient to intermittent intramuscular injection ; (ii) whether it was convenient for the medical officer; (iii) whether there was any ill effect from its use.

\section{Technique}

The instrument used was the Eudrip 2 (see Fig.), briefly referred to by McAdam, Duguid, and Challinor (1944). It is

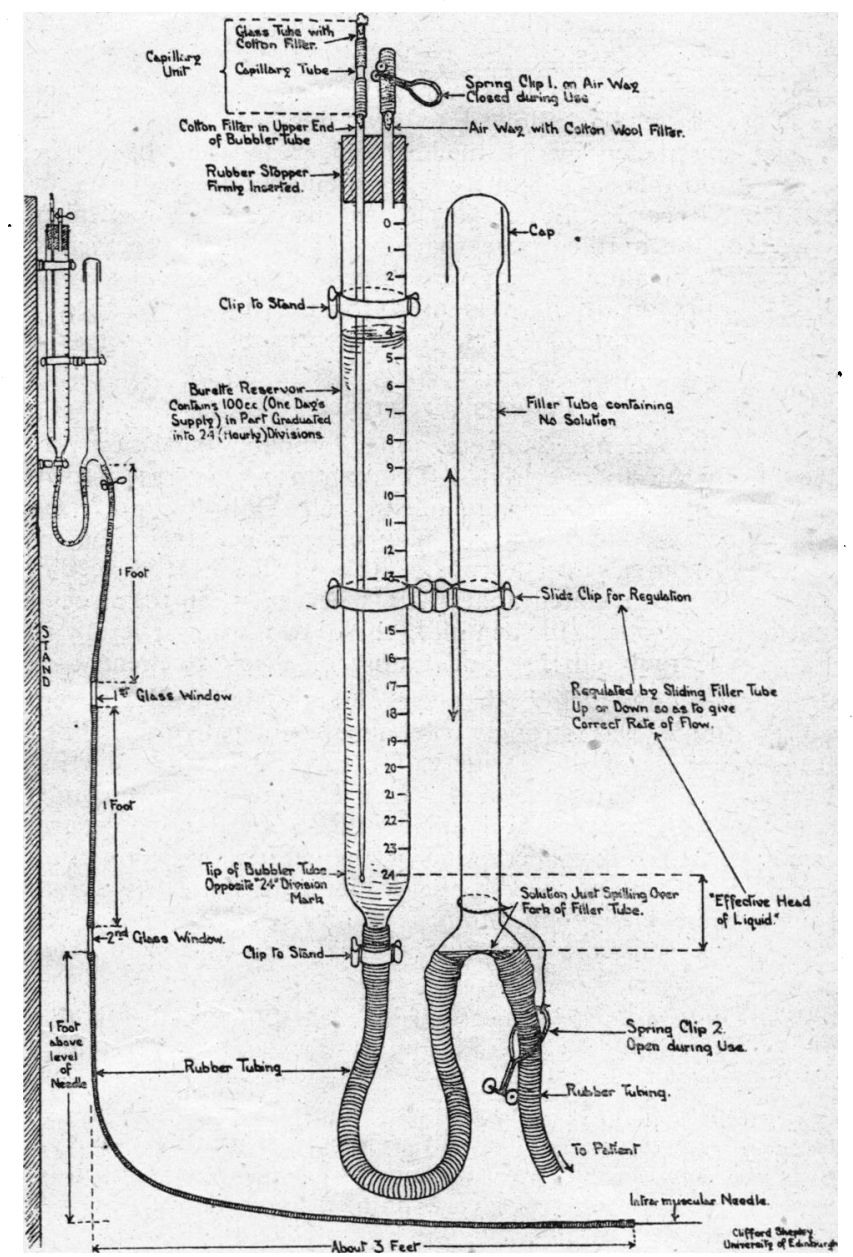

Eudrip apparatus No. 2 in use.

similar in principle to Eudrip 3, which they describe in detail, but instead of the 4-oz. "medical flat" bottle for reservoir there was a $100-\mathrm{c} . \mathrm{cm}$. burette with 24 graduations. In the top of the burette there is a rubber bung with two holes for the glass tubes. To the tube which passes to the bottom of the burette is attached the capillary air intake, to the other a piece of rubber tubing and a clip for release during filling. This is done by filling the side arm and elevating it. The side arm and other connexions are similar to those of Eudrip 3.

Three types of needle were used: the ordinary needle in an M.R.C. pattern blood-transfusion set; one similar to this but with two side perforations; a needle of the Middlesex pattern.

\footnotetext{
* In receipt of a grant from the Medical Research Council.
} 